\title{
La geología en las sociedades de cultura cientifica en el reinado de Isabel II. Francisco de Luxán profesor en la Sociedad de Instrucción Pública y en El Porvenir (1840-1848)
}

\author{
José María de Luxán Meléndez \\ jmluxan@cepc.es \\ Centro de Estudios Políticos y Constitucionales \\ Plaza de la Marina Española,9 28071 Madrid, España
}

\begin{abstract}
RESUMEN
Se presentan los caracteres institucionales de las sociedades de cultura científica en España durante el reinado de Isabel II, especialmente las de orientación progresista en las que Francisco de Luxán un científico, militar y político español del Partido Progresista, fue profesor de Geología en la Sociedad de Instrucción Pública y en El Porvenir entre 1840 y 1848. En las décadas centrales del XIX, junto al impulso de la ciencia desde el Estado es relevante la participación de la sociedad civil en el impulso de la ciencia mediante asociaciones culturales por sus actividades y por su organización, en un sentido amplio, pueden considerarse sociedades de cultura científica. La vinculación entre política, cultura y ciencia, medida por la participación poítica de los científicos y de los profesores de las sociedades culturales o por la repercusión en la prensa de sus actividades es muy importante.Y además la vinculación partidista permite calificar como sociedades progresistas la Sociedad de Instrucción Pública, y la sociedad El Porvenir. Las ciencias naturales, singularmente la enseñanza de la geología, tuvieron un papel destacado en las actividades y en la organización de estas asociaciones de cultura científica.
\end{abstract}

Palabras clave: Cultura científica, Francisco de Luxán, Política científica, Siglo XIX.

\section{Geology in scientific cultural societies in Spain under the reign of Isabel II. Francisco de Luxán professor at the Sociedad de Instrucción Pública and at El Porvenir society (1840-1848)}

\begin{abstract}
The institutional characteristics of the Spanish scientific cultural societies during the reign of Isabel II, especially those with a progressive orientation, are presented below. The Public Instruction Society and the EI Porvenir Society where Francisco de Luxán, a Spanish scientist, soldier and politician of the Progressive Party, was a Geology professor between 1840 and 1848.
\end{abstract}

Keywords: Francisco de Luxán, Scientific cultura, Scientific policy, $19^{\text {th }}$ Century.

\section{ABRIDGED ENGLISH VERSION}

2017 marked one hundred and fifty years after the death of Francisco de Luxán, a Spanish professor who first gave his "Geology Lessons" in 1840 at the Public Instruction Society and later in 1848 at the El Porvenir Society. Luxán (1799-1867) was a Spanish politician of the radical (or progressive) faction of Spanish liberalism, artilleryman in the Royal Academy of Sciences, chairman of the Geological Map Commission, member of the Spanish Parliament, senator, Minister of Public Works, State Councilor and member of the Bank of Spain's Board of Directors. 
The reign of Isabel II (1833-1868) was a time when many new political, economical and scientific institutions were developed. All these new institutions supposed an essential input for the development and consolidation of the liberal state and the new industrial society. In the scientific and technical field, this period is marked by the reconstruction and formation of a public place for science and technology. In the 1830s a new recovery phase and design of new scientific institutions occurred. These institutions, with different intensities and rhythms, were part of the new policies related to science and the industrial society that from the government's initiative gave a new model of public administration, new and recovered research and innovation public agencies, new public corporations and the introduction of science at the universities. In addition, thanks to the impulse of society it had new and recovered civil institutions.

From an institutional perspective, we use the category "science from society", to talk about all these nonstate organizations whose values and actions included a scientific orientation (information that scientists create, transmit, apply, etc.), and also an activity related to their contribution to the science popularization that refers to the part of science that all, or most, of the members of the society share, including those who are not scientists. This meant a perspective of the scientific culture as Quintanilla points out, which addressed a part of the culture that consists of the related information and consistent with scientific activity.

In the central decades of the nineteenth century, the impulse for science from society was expressed in the recovery of a model of enlightened institutions, which allowed the reconstruction of public corporations with associative bases such as the Academy of Sciences in Barcelona and the Societies of Friends of the Country. All these institutions were dissolved during the reign of Ferdinand VII but they got a new input during the first period of the reign of Queen Isabel II. During this period some new organizations such as the Academy of Sciences in Madrid appeared and it was also a moment of recovery for some other institutions such as the Ateneo of Madrid which was dissolved again during the moderate decade. Several new private organizations with a progressive orientation such as the Public Instruction Society were developed during the regency of the Espartero newspaper or the EI Porvenir Society in the moderate decade.

In this paper we use the term scientific cultural societies to emphasize an aspect of the activity and the organization of three cultural societies that, with more or less continuity, developed their activity in the central decades of the $19^{\text {th }}$ Century: the scientific, literary and artistic Ateneo of Madrid, the Public Instruction Society and the El Porvenir Society. An important part of their activity and their organization had a relevant scientific component and is evident in all three of them. They all had sections for natural sciences and exact sciences and their teaching of Zoology and Geology had a relevant position.

Because of the political orientation of its professors, the Ateneo de Madrid was identified as conservative but the Public Instruction Society and El Porvenir Society were widely seen as progressive. The contribution of Francisco de Luxán in progressive scientific cultural societies was hugely relevant. He was a Geology professor in 1840 and in 1848 he was also president of the section of Natural Sciences in the El Porvenir Society.

\section{La Sociedad de Instrucción Pública}

The Public Instruction Society was a civil association created in 1840 that promoted scientific culture and was a society with a progressive orientation in which politics and science were intimately linked. The objectives of the Society were to extend and provide useful insights to mankind. Geology appears as an imperative science that is sufficient in itself to fulfill a main influence on the wealth, well-being and future of our country (Luxán Miguel F., 1841).

Because of their impact on the press and their trajectory, the teaching programme included the Constitutional Law Course of Joaquín María López, Fermin Caballero s Geography Lessons and Francisco de Luxan s Geology Lessons.

\section{El Porvenir}

In 1847, El Porvenir, a scientific, artistic and literary society was created in Madrid. This project followed the main organizational guidelines of its predecessor, the Public Instruction Society. This included a teaching programme and the thematic sections which had been tested before in the conservative Ateneo de Madrid. This programme was at some distance from the Ateneo $s$ because it claimed a political and ideological commitment with the Progressive Party.

El Porvenir was divided into four sections: moral and political sciences; literature and fine arts; natural sciences and exact sciences applied to arts and crafts. The Natural Sciences section was headed by Francisco de Luxán. In this section many issues were discussed such as which of the three kingdoms of nature is more convenient to study, and which one would be more useful for Spain to develop. In the debate were two main competing issues. One was related with the theoretical idea of whether it was possible to debate, in the nat- 
José María de Luxán Meléndez, 2019. La geología en las sociedades de cultura... Boletín Geológico y Minero, 130 (2): $271-288$

ural science field, which kingdom was more important and if it was possible to rank the study of the mineral kingdom over the plant kingdom and this one over the animal or if it was better to do this in reverse. This idea added to the debate, according to El Espectador newspaper, the question of the pre-existence or the successive production of beings in nature. Secondly, was the question of which scientific field was more important to develop in Spain. According to Luxan, mineralogy should have a pre-eminent position.

The teaching plan included scientific, social science and humanities subjects. The newspaper Eco del Comercio writes about the geology lessons: Mr. Luxán has given a sample of what he is accredited for being; of deep political, of outstanding naturalistic, of accomplished philosopher, of eloquent speaker and a model of delicacy and finesse. The audience applauded such a beautiful speech in which we do not know what to praise more, whether the greatness of his knowledge or the brilliant way of exposing his ideas.

Because of the political events in Spain the activities of the society were suspended. After the suspension of the individual guarantees, El Espectador, in its 29 March 1848 edition, informed briefly that the Government had closed the El Porvenir Society.

In conclusion, in the central decades of the $19^{\text {th }}$ century, together with the promotion of the science given by the government participation of the civil society was also important. The promotion was given thanks to a generous impulse of science through the recovery of an enlightened institutional model that allowed, firstly, the reconstruction of public corporations with an associative basis such as the Societies of Friends of the Country or the Scientific Academies. Secondly, with associative forms of liberal values, which allowed the development of new and recovered cultural societies with a conservative or progressive orientation such as the Public Instruction society, during the Espartero regency, or the El Porvenir, in the moderate decade. According to their activities and organisation, as in the example of Ateneo de Madrid, they can be seen as Scientific Cultural societies.

The link between policy and science, measured by the participation of the Parliament or the Government, of scientists and members of the cultural societies or also by their repercussion in the press is very relevant and they can also been identified as progressive or conservative.

Natural science, especially Geology and the debate on the issue of the pre-existence or the successive production of beings in nature, became very important in the activities and the organisation of the progressive Cultural Science societies where Francisco de Luxan made a significant contribution. However, unlike Ateneo de Madrid, the progressive influence of the cultural science societies was mitigated due to their discontinuity and short life.

\section{Introducción}

El reinado de Isabel II que se inicia en 1833 es una etapa de diseño de nuevas instituciones políticas, económicas y científicas que van a contribuir al desarrollo y consolidación del Estado liberal y de la sociedad industrial. La alianza del trono y el pueblo que resume el programa de la Constitución de 1837, expresa la idea con la que los progresistas plasman el anhelo del buen gobierno. En el periodo de las regencias de María Cristina y de Espartero, que transcurre entre 1833 y 1843, la generación nacida en torno al ochocientos contribuyó a incorporar en la organización y en la acción del Estado una visión del progreso, cuyos objetivos de reformas políticas y económicas identifican con Europa, un marco institucional en el que se diseña y se pone en marcha una política pública para la ciencia y para la sociedad industrial. En la política para la ciencia en el reinado de Isabel II se pueden distinguir dos tipos de instituciones. En el ámbito de las instituciones estatales (la ciencia desde el Estado) desatacan la nueva administración pública liberal, el proyecto de universidad científica, las agencias públicas de investigación e innovación y las cor- poraciones públicas. Y en el ámbito de las instituciones civiles (la ciencia desde la Sociedad) nuevas y renovadas corporaciones públicas de base asociativa y sociedades culturales impregnadas por la política, cuyos objetivos, actividades y organización permiten, al menos en parte, entenderlas como sociedades de cultura científica.

Para abordar el estudio de las sociedades de cultura científica se señalan en primer lugar algunas de las características principales de la política científica en el reinado de Isabel II. A continuación se expone el concepto de cultura científica para aplicarlo a las sociedades culturales del tercio central del XIX, y se incorpora una semblanza de Francisco de Luxán, en la que se resalta su contribución a la política científica y a la política industrial. Y por último se estudian los objetivos, el programa de actividades, la estructura organizativa de estas sociedades y algunas características de la trayectoria política de sus profesores lo que permite resaltar en la generación del progreso la vinculación entre política, cultura y ciencia, así como la posición de la Geología en las sociedades de cultura científica de este periodo.

En este trabajo se presentan algunas característi- 
cas de las asociaciones constituidas para promover la cultura científica en España durante el reinado de Isabel II, especialmente sobre las de orientación progresista, en las que tuvo especial relevancia la Geología de la mano de Francisco de Luxán, del que en se cumplen, en 2017, ciento cincuenta años de su fallecimiento, y que fue un científico, militar y político español del Partido Progresista, que impartió sus Lecciones de Geología primero en 1840 en la Sociedad de Instrucción Pública y luego en 1848 en la sociedad El Porvenir, de la que fue presidente de la Sección de Ciencias Naturales.

\section{La política científica en el reinado de Isabel II}

López Piñero distinguió tres etapas en la ciencia española en el siglo XIX (López Piñero, 1992): la primera caracterizada por el colapso de la ciencia española, una etapa que denomina la catástrofe, y que transcurre entre 1808 y 1833; Una segunda, que corresponde a la España isabelina, que califica de intermedia, de 1833 a 1868; Y una tercera, entre 1868 y 1911, que llama la generación de sabios.

El reinado de Isabel II, estaría caracterizado por el inicio de un proceso de institucionalización de la ciencia, en el que a los científicos del tercio central del XIX se debió, por una parte la recuperación de los hábitos de trabajo científico, y la elevación del nivel de la información y la enseñanza; por otra, la creación de los grupos que actuaron de núcleos de cristalización de la actividad científica de la Restauración (López Piñero, 1992), una etapa en la que la vía de importación de conocimientos científicos se basa en una continuada política de becas, con la que en el ramo más favorecido, el de la minería y geología, salieron al extranjero unos treinta pensionados entre 1778 y 1850 (Vernet Gines, 1989).
El poder de la ciencia se afianzo en las décadas centrales del siglo XIX, al menos en Francia y en España, por el inicio del proceso de funcionarización, pero quizás lo más novedoso tenga que ver con que como nunca antes la ciencia y los científicos participaron en una esfera pública en continuo proceso expansivo $y$ animaron la construcción de una sociedad civil de la que procedía finalmente su legitimidad y autoridad como institución social (López-Ocón Cabrera, 2006).

Entre los políticos del progreso (Luxán Meléndez, 2016 a) Francisco de Luxán será el primero de una nómina muy reducida de ministros con formación científico-técnica, y entre los dirigentes del Partido Progresista y de la Unión Liberal será también el único con formación científico-técnica. En su conjunto entre los científicos el peso de los que participan activamente en la política es inicialmente muy alto (Luxán Meléndez,2016 b): un 9\% de los miembros de la Academia de Ciencias de Madrid son o serán ministros, y en el conjunto de las instituciones científicas, durante todo el periodo, la proporción de los son o serán parlamentarios es también muy alta (Tabla 1).

Una etapa de ensanche científico (López-Ocón Cabrera, 2003) que tendrá que ver con la política urbana, o con las necesidades de planificación de España, que estuvo íntimamente asociado al desarrollo de una política para la sociedad industrial, en la que la minería y el ferrocarril tuvieron un papel destacado. El periodo Isabelino, más que intermedio, tuvo un carácter no sólo de reconstrucción sino también de formación de un espacio público para la ciencia y la tecnología.

Es habitual situar entre los hitos fundacionales del proceso de institucionalización de la ciencia la creación en 1847 de la Academia de Ciencias Exactas, Físicas y Naturales, o en 1849 de la Comisión del Mapa Geológico, y en 1857 de la Facultad de Ciencias. Tres momentos, sin duda relevantes, que se sitúan en

\begin{tabular}{|l|c|c|c|}
\hline & $1836-1844$ & $1844-1854$ & $1854-1868$ \\
\hline & $\begin{array}{l}\text { Academia de Ciencias } \\
\text { Naturales de Madrid }{ }^{(1)}\end{array}$ & $\begin{array}{c}\text { Academia de Ciencias Exactas, } \\
\text { Físicas y Naturales }{ }^{(2)}\end{array}$ & $\begin{array}{c}\text { Agencias Científicas, de Innovación } \\
\text { y de Enseñanza superior }{ }^{(3)}\end{array}$ \\
\hline $\begin{array}{l}\text { \% de socios que son, } \\
\text { serán o han sido miem- } \\
\text { bros del parlamento }\end{array}$ & $30 \%$ & $23 \%$ & $20 \%$ \\
\hline $\begin{array}{l}\% \text { de socios que son, } \\
\text { serán o han sido ministros }\end{array}$ & $9 \%$ & $5 \%$ & $8 \%$ \\
\hline $\begin{array}{l}\text { (1) Incluye 32 académicos, (2) Incluye 55 académicos (3) Incluye 106 miembros de las siguientes instituciones: Comisión de } \\
\text { Estadística; Comisión Permanente de Pesos y Medidas; Observatorio Astronómico; Profesores de Escuelas de Ingenieros, Arquitectura } \\
\text { y Veterinaria de Madrid; Profesores de Ciencias en la Universidad de Madrid. }\end{array}$ \\
\hline
\end{tabular}

Tabla 1. Cientificos en la política en el reinado de Isabel II.

Table 1. Scientists in politics in the reign of Elizabeth II. 
un marco político de predominio conservador, en el que sin embargo no se suele identificar su carácter transversal en el que es fundamental la contribución de destacados científicos y políticos progresistas, de manera que esta visión tampoco tiene suficientemente en cuenta la agenda de institucionalización del progreso que impulsa el grupo extremeño (Luxán Meléndez, 2016 c) entre los que destacan Álvaro Gómez Becerra, José M ${ }^{\mathrm{a}}$ Calatrava, Antonio González, Facundo Infante y Francisco de Luxan.

En las décadas centrales del XIX, en España se impulsó una agenda pública en la que el progreso material derivado del conocimiento científico y causa del despliegue de la civilización moderna conducirá a un segundo espacio común, a la idea de fomento, concebida como la apuesta pública para activar el crecimiento del conocimiento y de la riqueza (PanMontojo, 2014).

Tras el colapso de 1823 , en la década de 1830 se inició una etapa de recuperación y de diseño de nuevas instituciones científicas que con distinto ritmo e intensidad, integran una política para de la ciencia y para la sociedad industrial (Luxán Meléndez, 2016 b) que desde la iniciativa del Estado, conto con un nuevo modelo de administración pública, con nuevas o recuperadas agencias públicas de investigación y de innovación, con nuevas corporaciones públicas, y es relevante destacar con la incorporación de la ciencia a la universidad. Y que además desde la la inicativa de la Sociedad también conto con nuevas o recuperadas instituciones civiles como las Academias de Ciencias Naturales de Madrid y Barcelona, las Sociedades de amigos del país, el Ateneo Científico y Literario de Madrid, la Sociedad de Instrucción Pública y la sociedad El Porvenir.

El desarrollo de la ciencia que se proyecta como un instrumento de articulación del Estado (LópezOcón Cabrera, 2003) y de la sociedad industrial, para los progresistas de 1837, está íntimamente asociado a las instituciones políticas liberales, a la libertad de pensamiento, de manera que la ciencia constituye el sólido y verdadero dique contra las demasías del poder (Llorente, 1836). Esta visión sobre el papel político de la ciencia, que incorpora un diagnostico extraordinariamente negativo de la situación de la ciencia española del primer tercio del XIX, es coherente con la explicación que sobre la situación social, económica y política construyen los liberales en Cádiz y que heredó el Partido Progresista.

Una situación de infortunio que también desde la Academia de Ciencias de Madrid se atribuye al mal gobierno de la monarquía absoluta, a la ruptura de las antiguas instituciones de Castilla y Aragón, favorables a las libertades públicas, protectoras de las ciencias (Llorente, 1836). Una posición arraigada en la Academia desde el discurso inaugural de Antonio Ortiz de Traspeña que dirá: Nada puede prometerse la sabiduría donde la libertad civil esta sofocada. Y si desde la Academia se asocian las posibilidades de desarrollo científico a la consolidación de las instituciones liberales, en las Cortes, Francisco de Luxán, añadirá que el conocimiento es el único sostén del mérito (DSCC 16 de diciembre de 1836).

\section{Cultura científica}

La expresión cultura científica (Quintanilla Fisac, 2010) tiene un carácter polisémico, puede emplearse como equivalente a ciencia, y estaría formada por la información que crean, transmiten, aplican, etc. los científicos; también se utiliza para referirse a la parte de la ciencia que comparten todos (o la mayoría) de los miembros de una sociedad, aunque no sean científicos; y además se puede aludir a una parte de la cultura que consiste en información relacionada y compatible con la actividad científica, aunque como tal no forme parte de la actividad científica, y en la que se podría a su vez distinguir entre la cultura de los científicos, es decir las creencias, reglas de actuación y valores científicos propiamente dichos, y en un sentido extenso las creencias, reglas de comportamiento $y$ valoraciones referidas a la ciencia y compatibles con ella pero que no forman parte de la ciencia.

En este trabajo, desde la perspectiva del institucionalismo, utilizamos la categoría de la ciencia desde la sociedad para referirnos a organizaciones no estatales cuyos valores y actividades incluyen una orientación científica propiamente dicha (información que crean, transmiten, aplican, etc. los científicos), y al tiempo una actividad orientada a la divulgación científica (que equivale a la parte de la ciencia que comparten todos (o la mayoría) de los miembros de una sociedad, aunque no sean científicos, lo que supone una perspectiva de cultura científica (referida a una parte de la cultura que consiste en información relacionada y compatible con la actividad científica)

Estas instituciones sociales, cuyos valores y actividades forman parte de la cultura científica, fueron de dos tipos: Corporaciones públicas de base asociativa y Sociedades privadas.

En las décadas centrales del XIX, el impulso de la ciencia desde la sociedad se expresó en la recuperación de un modelo de instituciones ilustradas, que permitió la reconstrucción de corporaciones públicas de bases asociativa como las Sociedades de Amigos del País, y las Academias científicas, disueltas en el periodo fernandino y que en el reinado de Isabel II 
tendrán una segunda vida. Y además de la recuperación del Ateneo de Madrid, disuelto igualmente en la década ominosa, se desarrollaron nuevas sociedades de orientación progresista tales como la Sociedad de Instrucción Pública, durante la regencia de Espartero, o El Porvenir en la década moderada (Tabla 2).

\section{Semblanza de Francisco de Luxán}

Francisco de Luxán Miguel-Romero (1799-1867) fue un político progresista, artillero, de la Real Academia de Ciencias, presidente de la Comisión del Mapa Geológico, diputado, senador, ministro de Fomento, consejero de Estado y miembro del Consejo de Administración del Banco de España (figura 1).

Luxán forma parte del grupo de liberales extremeños que apoyaron primero a Calatrava, luego a Espartero y más tarde a O`Donnell. Entre 1833 y 1868 los progresistas extremeños (Luxán 2016 c) conforman una corriente política de carácter transversal, primero encuadrados dentro del Partido Progresista y luego en la Unión Liberal. Un grupo que cuenta con una agenda política propia, que propugna el progreso legal y la unión de los liberales y que es visto de forma homogénea por la prensa y por los otros actores políticos, que les identifican con Espartero.

Mariscal de Campo en 1863, Luxán fue un artillero de las promociones itinerantes, que asistió a la derrota delTrocadero en 1823, y depurado del ejército en la década ominosa, se reincorporó al servicio activo entre 1833 y 1843 . En la guerra civil, la de los siete años, siendo diputado, fue en 1837 oficial de enlace entre Espartero y los generales Evans, y Sarsfield. Recibió la Cruz Laureada de San Fernando.

Desde la Fundición de Bronce de Sevilla, a la que en 1827 se incorporó impurificado, pasando por la cárcel en 1831 y por una etapa de formación científica en Madrid, en la cátedra de Química Docimástica que en la Dirección General de Minas impartio José Duro y Garcés, y en la cátedra de mineralogía de Donato García en el Gabinete de Historia Natural, y en París, en la Escuela de Minas, Luxán, durante la minoría de edad de la reina, fue profesor de Isabel II,

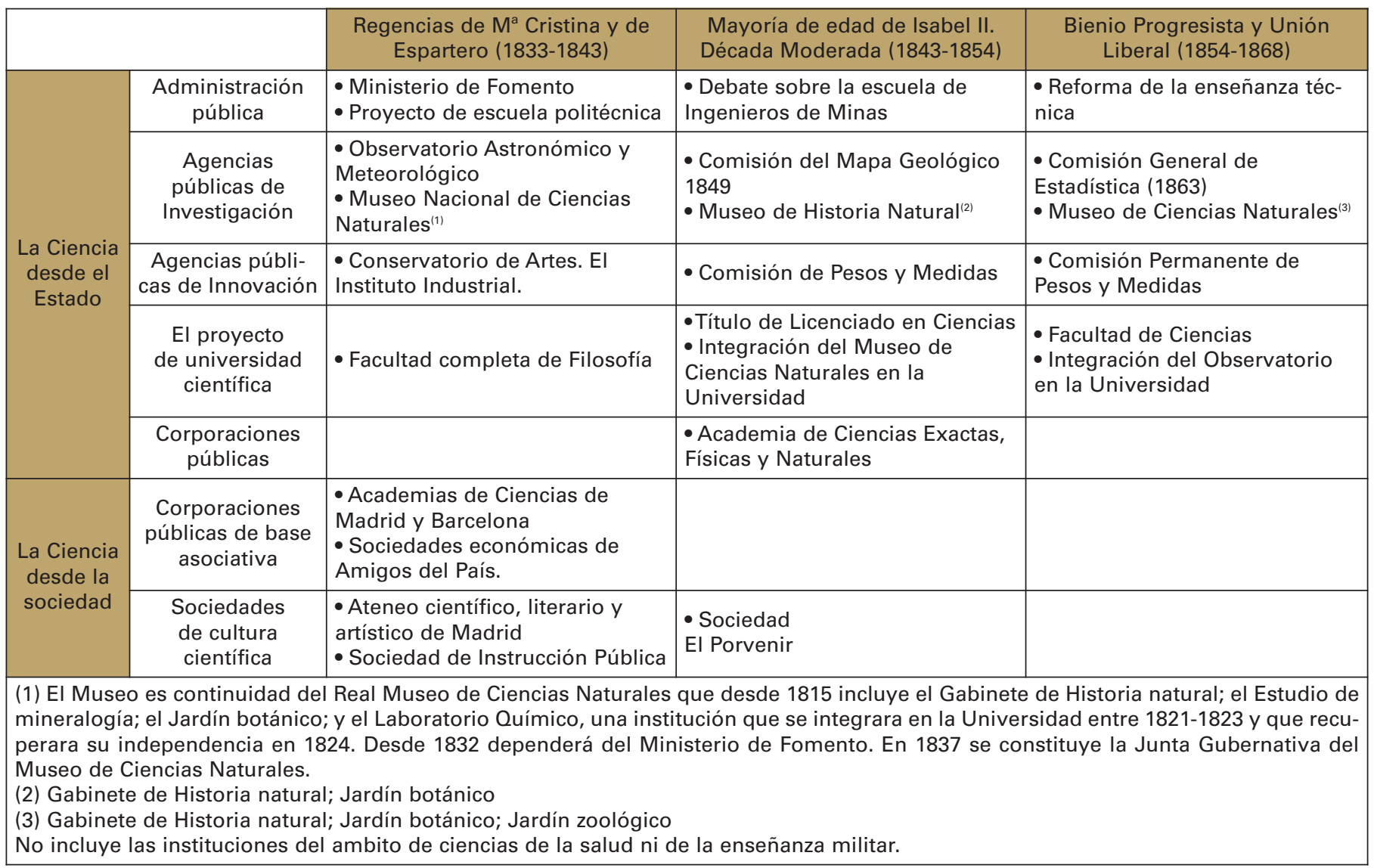

Tabla 2. Instituciones científicas (1833-1868).

Table 2. Scientific institutions (1833-1868). 
y estuvo destinado en la secretaría de Guerra, y en la de Estado.

Vicepresidente de la Real Academia de Ciencias Exactas, Físicas y Naturales en 1866, fue autor de numerosas monografías científicas y tecnológicas, miembro de varias sociedades y corporaciones científicas nacionales e internacionales, Luxán tendrá una posición destacada en las agencias públicas de investigación y de innovación de las décadas centrales del siglo XIX.

En los años siguientes, hasta 1867, su trayectoria científico-técnico continuó en ascenso; sucesivamente fue presidente de la Sección de Ciencias Naturales de la sociedad El Porvenir, presidente de la Comisión del Mapa Geológico, en la que tendrá una participación decisiva (Rabano Gutierrez del Arroyo, 2015 y 2016), director de Operaciones Geodésicas de la Comisión General de Estadística (Muro, J. I., Nadal, F., \& Urteaga, L. 1996), comisario regio del Observatorio Astronómico, y presidente de la Comisión de Pesos y Medidas.

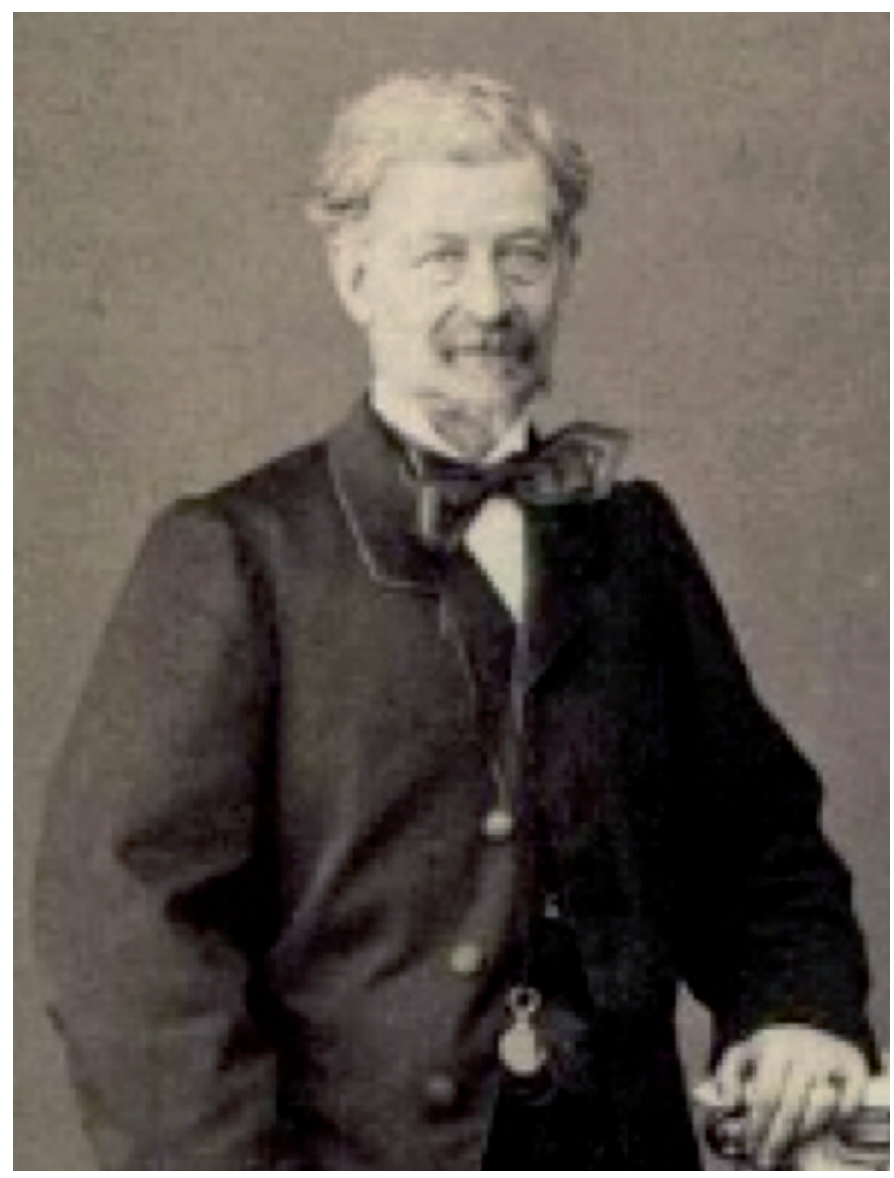

Figura 1. Francisco de Luxán Miguel-Romero (Fotografía Jean Laurent).

Figure 1. Francisco de Luxán Miguel-Romero (Photography Jean Laurent).
Entre 1833 y 1844, durante las regencias, ingresó en la Academia de Ciencias Naturales de Madrid, en la Sociedad Geológica de Francia, en la Sociedad Matritense de Amigos del País, en la Sociedad Económica Constantinense de Amigos del País, en el Instituto Industrial de España, y en la Sociedad de Instrucción pública. Imprime su itinerario europeo, e imparte sus lecciones de Geología. Y recibe el encargo de preparar un tratado de Mineralogía para la Academia de Artillería, de la que fue profesor de Química.

Ministro de Fomento primero con Espartero en el bienio progresista, y luego con O'Donnell al terminar el gobierno largo, Luxán contribuyó a conformar una política para la sociedad industrial en la que la minería, el ferrocarril y el fomento de la industria serán tres de los argumentos que conforman la agenda pública. Una política de impulso de agencias de innovación que se desarrolló paralela a la política para la ciencia, que se expresa mediante agencias públicas de investigación, el impulso de la universidad científica y el desarrollo de nuevas y recuperadas sociedades y corporaciones científicas.

Miembro de la Junta Central del Partido Progresista, y de la dirección de la Unión Liberal. Diputado por Badajoz, Baleares, Madrid y Santander. Senador Vitalicio. Consejero de Estado, Luxán fue redactor del periódico progresista El Espectador y de la revista también progresista La España Militar.

\section{Las Sociedades de Cultura científica}

En este trabajo utilizamos la expresión sociedades de cultura científica para subrayar un aspecto de la actividad y de la organización de tres sociedades culturales que con mayor o menor continuidad desarrollaron su actividad en las décadas centrales del XIX: EI Ateneo científico, literario y artístico de Madrid, la Sociedad de Instrucción Pública y la sociedad EI Porvenir (Tabla 3).

En las tres una parte de su actividad y de su organización tienen un componente científico significativo. La mitad de sus secciones son de orientación científica y una proporción importante de sus cátedras son también científicas. Cuentan con secciones de ciencias naturales y de ciencias exactas, en las que entre otros se debaten temas de relevancia científica como la cuestión de "la preexistencia o sucesora producción de los seres en la naturaleza" (El Espectador 19/01/1848) y en su docencia incorporaron la Física, la Química, las Matemáticas, la Zoología y la Geología.

Por la orientación política de sus profesores y por sus objetivos se identifica como conservador, el 
José María de Luxán Meléndez, 2019. La geología en las sociedades de cultura... Boletín Geológico y Minero, 130 (2): $271-288$

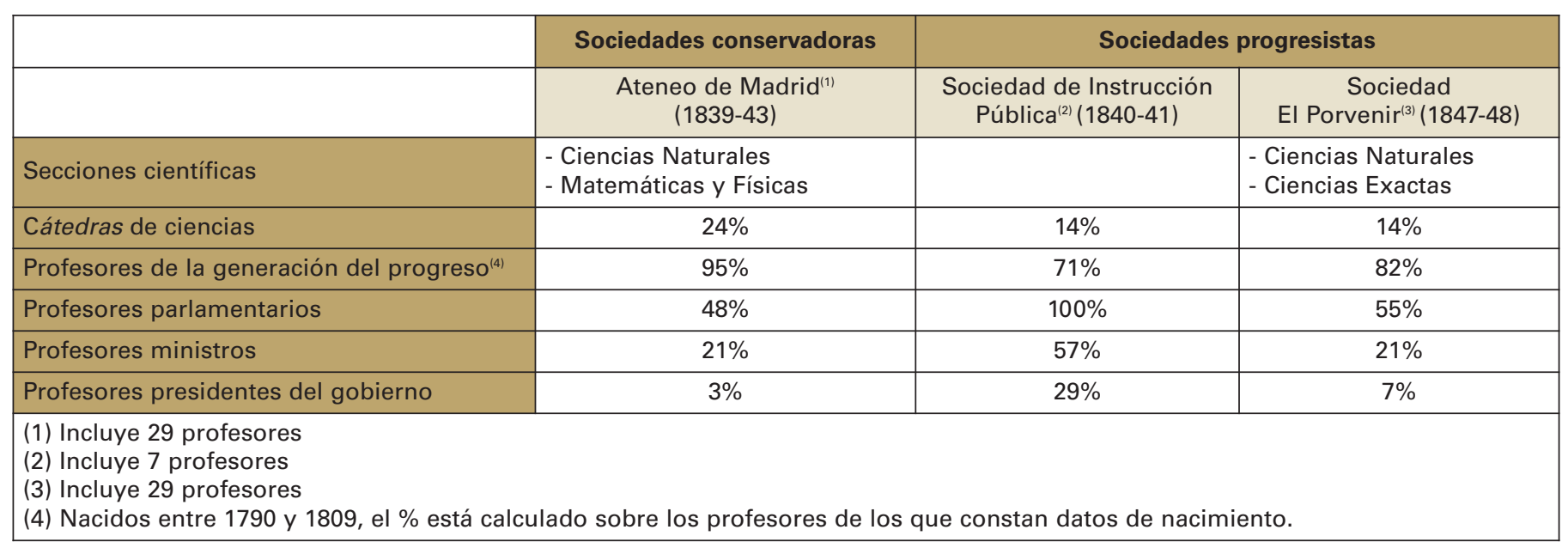

Tabla 3. Ciencia y política en las sociedades de cultura científica.

Table 3. Science and politics in the societies of scientific culture.

Ateneo de Madrid y como progresistas la Sociedad de Instrucción Pública, y la sociedad El Porvenir. Las sociedades de orientación progresista y conservadora en gran medida son compartimentos estancos que no comparten el profesorado. Sólo tres de los profesores del Ateneo, Alfredo Camus, Fernando Corradi y Pedro Gómez de la Serna, serán también profesores en las sociedades progresistas, y por ejemplo Francisco de Luxán, profesor en las dos progresistas declinará expresamente la invitación que recibió en 1852 para dar un curso de Geología en el Ateneo.

Los profesores de las tres sociedades tuvieron una actividad política muy relevante, gran parte de ellos fueron diputados y un número muy relevante formaron también parte del gobierno, cuatro de ellos; Juan Francisco Pacheco, Luis González Bravo, Joaquín María López y Salustiano Olózaga fueron además presidentes del Gobierno.

Tanto en las Sociedades progresistas como en el Ateneo los profesores y los miembros de las Juntas de Gobierno, casi en su totalidad, pertenecen a la generación que alcanza y desarrolla su madurez política, social, económica e intelectual durante el reinado de Isabel II, y que conforma un cuerpo generacional homogéneo cuyos primeros años tienen como punto de arranque la Revolución Francesa (1789) y como límite superior su traslación a España con la Declaración Revolucionaria de Cádiz (1810), con la que las Cortes nacionalizan la soberanía. Esta generación nacida en torno al ochocientos que denominamos del progreso (Luxán Meléndez J. M., 2016 a) incluye a los nacidos entre 1790 y 1809.

La continuidad del Ateneo contrasta con la discontinuidad de las sociedades progresistas, cuya consolidación se verá alterada por los vaivenes del sistema político y finalmente serán clausuradas en 1848 en la deriva autoritaria de Narváez. Aunque, el 26 de marzo de 1848 varios periódicos anuncian que el lunes 27 en El Porvenir continuará Francisco de Luxán con sus lecciones de Geología, los acontecimientos políticos lo impidieron y tras la suspensión de las garantías individuales, El Espectador en su edición del 29 de marzo escuetamente informó que el Gobierno mediante un oficio del jefe político de Madrid clausuró El Porvenir, y unos días más tarde, el 11 de abril, se hace eco de que la fiscalía militar solicitó su lista de socios, y también la de la clausurada sociedad progresista Tertulia del 18 de junio.

“Esta Academia, compuesta en su mayor parte de notabilidades literarias y políticas, circunstancia que la aseguraba larga existencia, hubiera sin duda producido lucidísimos y ventajosos resultados entre la juventud estudiosa que se afanaba por oír las lecciones de hombres eminentes, si un acontecimiento lamentable no hubiese suspendido la enseñanza a consecuencia de una orden expresa del Gobierno. De desear es que se abran de nuevo las cátedras y que se lleve adelante el pensamiento que se propusieron los hombres distinguidos que fundaron y protegieron esta Academia"(Madoz, 1845-1850). Este deseo que expresó Madoz en su Diccionario no se cumplirá.

\section{Las sociedades conservadoras: El Ateneo Científico y Literario de Madrid}

En octubre de 1835 la Sociedad Matritense de Amigos del País auspicia la creación de un Ateneo Científico y Literario a imagen del que funcionó en Madrid entre 1820 y 1823 , y en el que la teoría de la monarquía libe- 
ral (Garrorena Morales, 1974) y en general las ideas conservadoras encontrarán un espacio privilegiado desde el que orientar las instituciones políticas.

En el ámbito científico en sus cátedras, entre 1839 y 1841, Manuel López Santaella impartirá un curso de Geología, Juan Mieg, del Real gabinete de físicaQuímica, impartirá un curso de Zoología, Venancio González Valledor, luego de la Academia de Ciencias, impartirá un curso de Física, Alfredo Adolfo Camus de Matemáticas, José $\mathrm{M}^{\mathrm{a}}$ Nieva de Química aplicada a la agricultura, Jaime Salvá de Fisiología general y humana y los académicos de la de Ciencias Naturales de Madrid, Leoncio Sobrado y Eusebio $\mathrm{M}^{\mathrm{a}}$ del Valle, respectivamente de Fisiología y de Economía Política (Tabla 4).

El Ateneo estaba organizado en cuatro secciones: Ciencia Morales y Políticas; Ciencias Naturales; Ciencias Matemáticas y Físicas; y Literatura y Bellas Artes. En el ámbito de las secciones científicas intervinieron José Francisco Aizquivel (1798-1865), con-

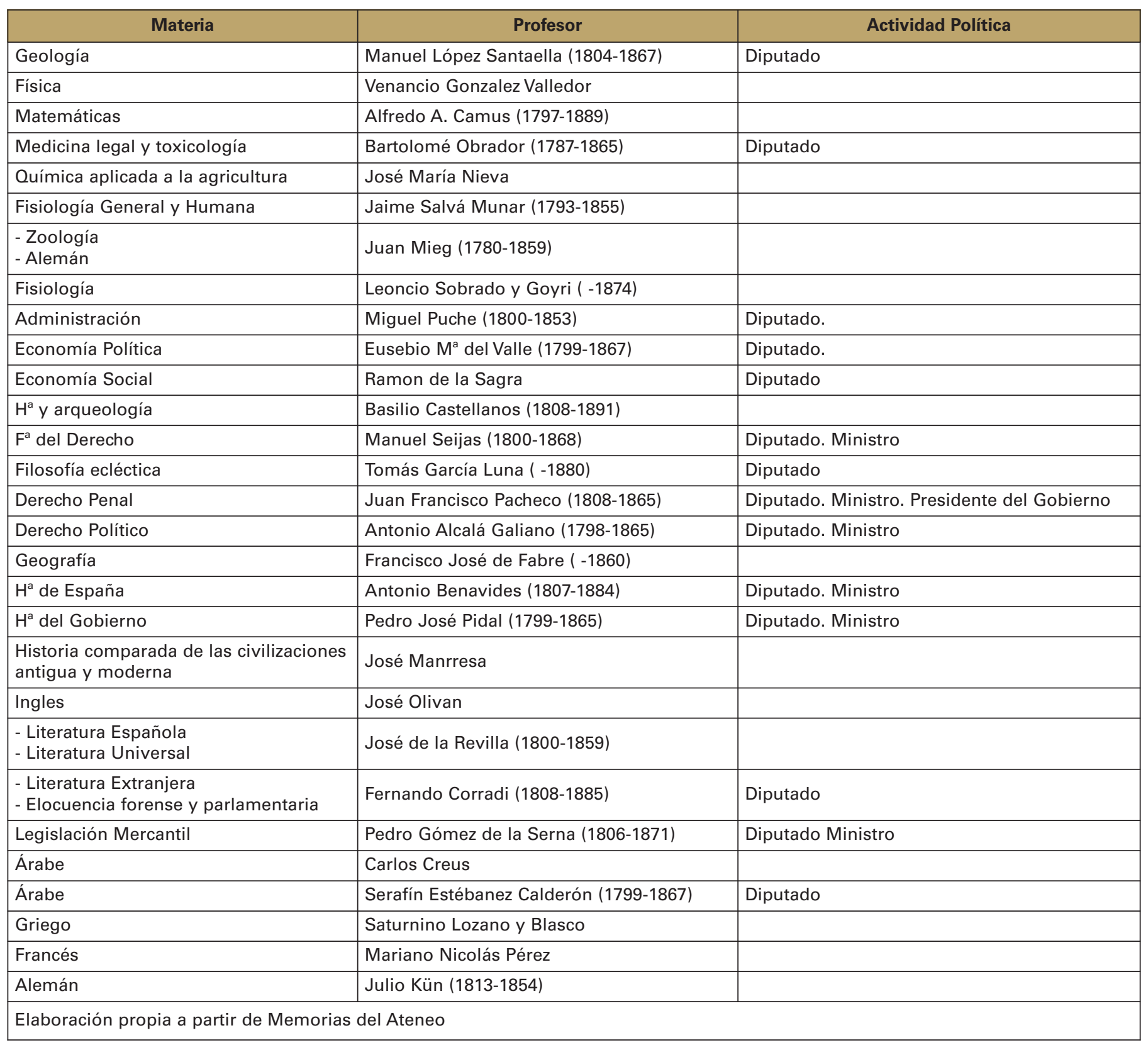

Tabla 4. Profesores del Ateneo científico y literario de Madrid (1839-1843).

Table 4. Professors of the scientific and literary Athenaeum of Madrid (1839-1843). 
servador perpetuo del Gabinete de Física é Historia natural que leyó un extracto razonado de la obra titulada "Historia Natural agrícola y económica del Maíz", publicada por Mateo Bonafons en 1835 (Monreal, 1839), Antonio Tenreiro (1792-1855), fue presidente de la sección ciencias naturales del Ateneo, explico el influjo de estas ciencias en la prosperidad y riqueza de la naciones (Monreal, 1839), Rafael Cabanillas (1778-1853) fue director general de Minas, realizó un Examen de los muchos y ricos criaderos o depósitos de carbón piedra que abriga el suelo español (Monreal, 1839) Benito del Collado y Aradnuy, inspector general del Cuerpo de Ingenieros de Minas, presentó una reseña de la riqueza mineral plomiza que se beneficia España (Monreal, 1839) y José Mariano Vallejo (1779-1846) presidente de la sección de Ciencias Matemáticas y Físicas del Ateneo presentó una memoria en que se trata de algunos puntos relativos al sistema del mundo y formación del globo terrestre en que habitamos; con aplicación a investigar nuevos procedimientos para facilitar la separación y aprovechamiento de la plata que contiene el plomo, y determinación de los parajes en que hay más probabilidades de que existan minas de plata en la Sierra Nevada de Granda, y de cualquiera otros metales en el resto de la Península (Monreal, 1839)

\section{Las sociedades progresistas: La Sociedad de Instrucción Pública y la sociedad EI Porvenir.}

En 1840, la Sociedad de instrucción pública, y en 1847, su sucesora El Porvenir son entidades civiles de orientación progresistas formadas entre otros objetivos para promover la cultura científica, son instituciones constituidas "con el fin de conjugar la enseñanza de las materias útiles con lo que hoy llamaríamos ciencias sociales" (Elorza, 1987).

En ambas sociedades política y ciencia se muestran íntimamente vinculadas. Gran parte de los profesores de ambas sociedades, o de los miembros de su Junta de Gobierno y de sus secciones fueron destacados miembros del Partido Progresista.

Los objetivos de la Sociedad de Instrucción públi$\mathrm{ca}$, que desarrollo su actividad durante la regencia de Espartero, eran extender y propagar los conocimientos útiles al género humano, entre los cuales la geología aparece como una ciencia indispensable destinada a ejercer un influjo determinante en la riqueza, en el bien estar $y$ en el porvenir de nuestro país (Luxán Miguel-Romero F. , 1841).

En noviembre de 1840 la Sociedad de Instrucción Pública publicó un prospecto en el que detalla sus objetivos.
"Conociendo varios ciudadanos amantes de la prosperidad de España que uno de los objetos más dignos de la inmediata solicitud de todo buen patriota es la educación general, única fuente de moralidad, tolerancia y progreso, determinaron crear una sociedad, que, sin más designio que el de la pública y gratuita enseñanza, pudiera, con ayuda del tiempo y de sus desvelos, hacerse., algún día digna de la gratitud del país. En la actualidad más que nunca debe de resultar fecunda una idea, por suyo medio al mismo tiempo que se procura cultivar la inteligencia del pueblo, se facilita la futura acción de aquellos gobiernos justos y reformadores que con tanto escándalo reclaman los abusos presentes". (El Eco del Comercio 28 /11/ 1840 y la Gaceta de Madrid 8/12/ 1840)

El Porvenir fue una academia de ciencias, literatura y bellas artes, que desarrolló su actividad entre junio de 1847 y abril de 1848, es un proyecto que, como él de su antecesora Sociedad de Instrucción Pública, sigue las pautas institucionales ensayadas en el Ateneo, pero se distancia de este porque de manera expresa se reclama política e ideológicamente comprometido con el partido progresista. El Porvenir manifestó un interés en la difusión del conocimiento, “cuya propagación había monopolizado hasta ahora en provecho de sus miras particulares el partido de la suprema inteligencia" (El Espectador se refiere al partido moderado) Se propone impulsar "las buenas doctrinas científicas, literarias y artísticas que más puedan contribuir a los progresos de la civilización y de la felicidad del país". Y tiene voluntad de cumplir un objetivo de formación de los cuadros del partido progresista, de forma que en sus salas "ya lucen sus elevados talentos y sus dotes de buen decir, dignos presagios de la misión que están llamados a desempeñar en la administración del estado y en el seno del parlamento" (El Espectador. 6/02/1848).

El ateneo de los progresistas, tanto en las reuniones de sus secciones como en sus cátedras se propuso actuar en dos ámbitos. En sus secciones se discute sobre algunos temas que impregnan la cultura científica y sobre la orientación de las políticas públicas de las décadas centrales del XIX. La evolución, el sufragio universal, el papel del estado en la economía, o la vinculación entre la política científica y la política económica son alguno de los temas de debate. $Y$ en sus cátedras se inició un programa de enseñanza multidisciplinar que incorporó aéreas de interés político, cultural o científica: En el campo científico, materias como la geología o la higiene pública. En las ciencias sociales: estadística, economía política, o socialismo.Y en letras: estética o bellas artes. Además pondrá en marcha una oferta de enseñanza de lenguas modernas: francés e inglés. 


\section{Organización}

La actividad que la Sociedad de Instrucción Pública desarrolló en Madrid se reflejó casi diariamente en los periódicos progresistas, en la Gaceta y en el Diario de avisos de Madrid.

"Vencidas ya todas las dificultades, el domingo 29 de noviembre de 1840 se abrirá el nuevo establecimiento y desde $1^{\circ}$ del próximo diciembre darán principio las enseñanzas. Se financia con donativos voluntarios de algunos patriotas respetables han secundado las miras de la sociedad contribuyendo á allanar en breve término obstáculos materiales...La entrada estará franca para el público. Las personas que gusten cooperar á la realización y desarrollo de este proyecto podrán suscribirse en concepto de socios, mediante la entrega de $10 \mathrm{rs}$. mensuales y adelantados; para cuyo efecto acudirán al despacho de don José Vidal, tesorero de la sociedad" (El Eco del Comercio).

El Porvenir realizó su actividad también en Madrid (El Espectador 28/27/1848 o el Diario Oficial de Avisos de Madrid 26/11/1847 y 9/1/1848), en el principal del número 15 de la carrera de San Jerónimo en un local en el que "estaba suntuosamente decorado el magnífico salón de conferencias adornado con alfombra, butacas, espejos, quinqués y otros objetos elegantes y exquisitos lo mismo debe decirse de las piezas destinadas para la enseñanzas y otras ocupadas con las dependencias del establecimiento" (Madoz, 1850).

La sociedad escribe Madoz, dos año después de su fundación, estaba formada "por amantes de la ilustración, protectores decididos de las clases menos acomodadas del pueblo, (que) trabajaron asiduamente con el objeto de proporcionar al público una enseñanza gratuita, digna de la capital de España (y) para subvenir a las atenciones de la sociedad, sus individuos pagaban mensualmente una cuota módica".

El 19 de enero de 1848 se inauguraron las cátedras del Porvenir, "la concurrencia fue escogidísima y superior con mucho a la que puede contener de suyo aquel bello y elegante local, quedando más y más realzada la solemnidad del acto con la asistencia del bello sexo, el cual tiene destinada para sí una parte preferente del salón en que se verifican las explicaciones". Como en su antecesora, Sociedad de Instrucción Pública, también en El Porvenir en la inauguración "pronunció uno de sus más bellos discursos el elocuente señor Don Joaquín María López" (Madoz, 1845-1850). El discurso enfatiza EI Espectador "fue como todos los suyos un modelo de arrebatadora elocuencia" (El Espectador 20 de enero de 1847).

El Porvenir cuenta con una Junta General, en la que pueden participar todos los socios, que se reunirá de manera ordinaria o extraordinaria, y con mayor asiduidad lo hará la Junta Directiva y las comisiones creadas por ella (entre otros Diario Oficial de Avisos de Madrid 26 de noviembre de 1847 y 9 de enero de 1848 o El Espectador 17, 20 y 26 de febrero 1848 o del 11de marzo)

Desde el comienzo de sus actividades la prensa progresista (Por ejemplo El Espectador en el mes de enero de 1848 los días 12, 13, 18, 19, 20, 21, 22, y 28, en el mes de febrero de 1848 los días 3, 6, 9, 10,11,15, $17,18,19,20,24,25,26,27$, y en el mes de marzo los días $1,10,11,12,14,18,19$ y 29 ) incluirá información sobre sus conferencias, reuniones y debates, y además se mostrará muy satisfecha con "la nueva sociedad científica, literaria y artística que se ha fundado hace poco y que se va organizando de una manera que no hay más que pedir; como que en el poco tiempo que lleva de vida cuenta ya con un gran número de socios, entre los que figuran las primeras notabilidades políticas y literarias de España, que inspiradas por un celo altamente patriótico se prestan a instruir gratuitamente al pueblo en las ciencias y artes de más útil y constante aplicación" (El Tío Camorra 10 de noviembre de 1847).

\section{Programa de actividades docentes}

En noviembre de 1840 la Sociedad de Instrucción Pública (El Eco del Comercio 28 /11/ 1840 y la Gaceta de Madrid 8/12/ 1840) anunció que desarrollaría siete materias, entre las que figura la Geología. $Y$ en diciembre de 1847 el Porvenir anunció un programa mucho más ambicioso compuesto por 31 cátedras encomendadas a destacados progresistas -entre otros El Eco del Comercio, El Español, y El Heraldo 8/12/1847- (Tabla 5)

Por su impacto en la prensa y por su posterior publicación, en el programa de enseñanza destacan el Curso de Derecho Constitucional de Joaquín María López (López, 1840), las lecciones de Geografía de Fermín Caballero (Burgueño, 1994) y las Lecciones de Geología de Francisco de Luxán (Luxán F., 1841).

El Plan docente de 1847 como el de 1840, se desarrollaría también de de lunes a domingo, entre las seis y las nueve de la noche, pero en vez de una lección será con tres cátedras diarias, cada una con una hora de docencia. Se incluyen materias científicas, de ciencias sociales y de Humanidades.

En ambas sociedades los profesores impartirán las clases gratuitamente y en algunas materias la asistencia será libre y en otras los alumnos deberán matricularse. 


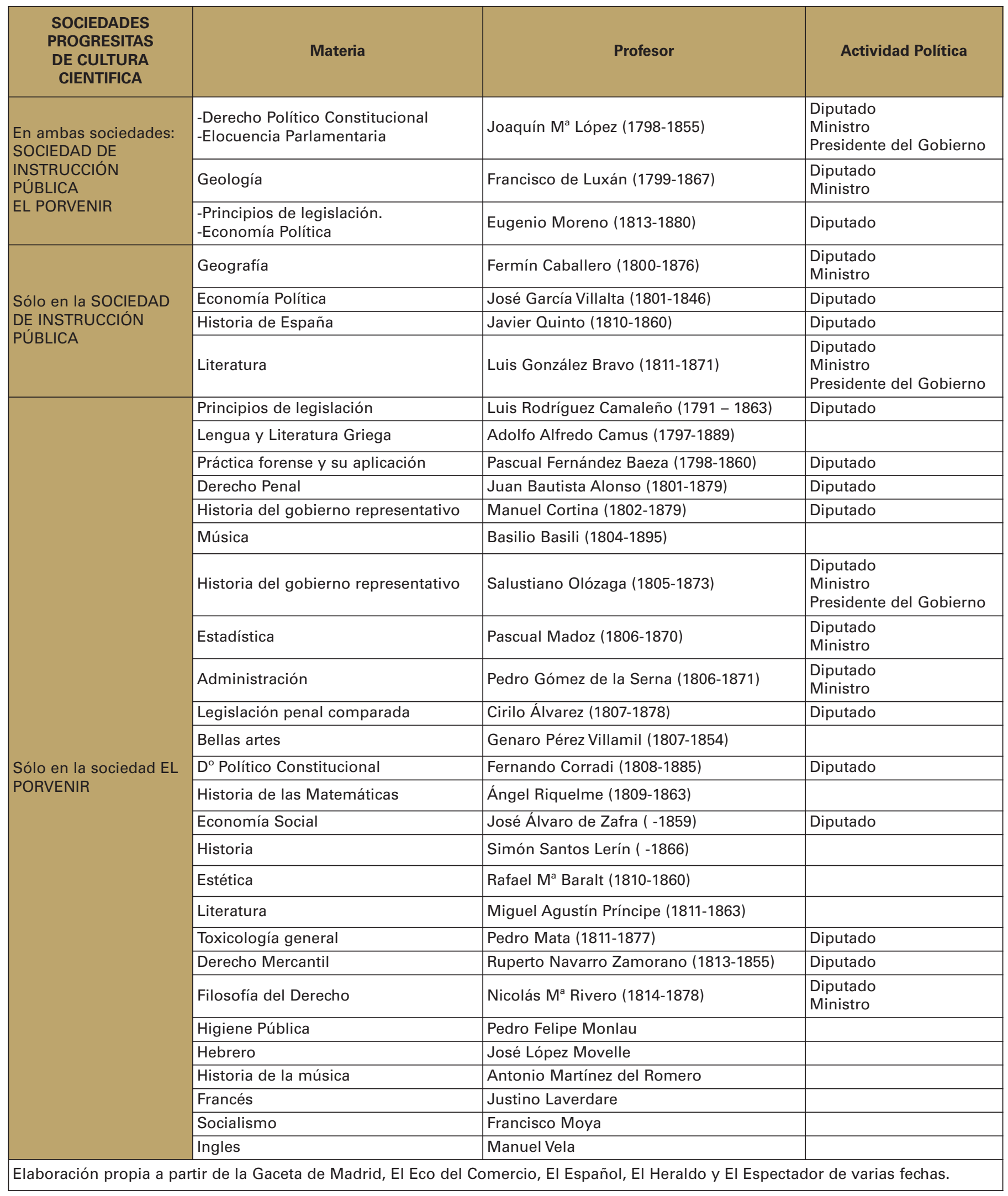

Tabla 5. Los profesores de las sociedades progresistas de cultura científica.

Table 5. Professors of progressive societies of scientific culture. 
Para los progresistas el balance inicial no puede ser más satisfactorio:

"La juventud, ansiosa de saber, no ha cesado una sola noche de agolparse afanada a sus puertas, adelantándose con mucha anticipación á las horas que se han fijado para las explicaciones, temerosa de hallar ocupado aquel bello y elegante recinto, donde tan alta muestra dan de si nuestros hombres de ciencia y doctrina en la mayor parte de los conocimientos que constituyen el saber humano... El Porvenir no basta a contenerla escogida y numerosa concurrencia que se afana por oír los debates, donde tantos brillantes jóvenes esperanza de nuestro partido lucen sus elevadas talentos y sus dotes de buen decir, dignos presagios de la misión que están llamados a desempeñar en la administración del estado y en el seno del parlamento.

Pobre y proscrito el bando del progreso, es rico en virtudes y en ciencia. Esos jóvenes se han formado en la persecución y en la desgracia. A los hombres que les han precedido, y cuya conducta ejemplar les sirve de norte, les ha sucedido lo propio. El anatema y la tribulación que pesan sobre sus cabezas, no han sido poderosas a amortiguar en ellas la ardiente fe de su corazón, ni el fuego de la inteligencia.

El pensamiento de erigir en España un nuevo templo a las ciencias a las letras y a las artes reunidas, obra ha sido de la juventud que el progreso encierra en su seno. Los ancianos de ese mismo progreso lo han coronado aceptándolo y adhiriéndose a él completamente. Hombres de tradición y hombres nuevos, obedeciendo todos a una sola e idéntica inspiración, se afanan en llenar dignamente la misión del profesorado, formando parte de él hasta los misinos que en nuestras disensiones anteriores aparecieron momentáneamente divididos, no en los principios que a todos eran comunes, sino en la apreciación de algunos hechos susceptibles de ser comprendidos de muy distinta manera. Hermoso es y consolador el aspecto que desde este punto de vista presenta la nueva academia. Los que hablando solo por baldar suponen todavía existentes nuestras desavenencias pasadas, o creen tal vez, en la fábula de es otra discordia incipiente, entre los jóvenes y los llamados viejos. Lean la lista de los profesores que figuran en EI Porvenir los nombres de López, la Serna, Olózaga, Luxan. Cortina, Mata, Alonso, Corradi, Lerin, Camareño, Moreno López, Madoz, Villergas, Príncipe, Camús, Baeza, Rivero, Baralt, Navarro, Zamorano y demás catedráticos que comparten entre sí la tarea de difundir las buenas doctrinas científicas, literarias y artísticas que más puedan contribuir a los progresos de la civilización y de la felicidad del país, serán á semejantes imposturas la mejor y más cumplida respuesta" (El Espectador 6 de febrero de 1848)
Las lecciones de Geología de Francisco de Luxán

Las lecciones de Geología publicadas en 1841 (Figura 2) que Luxán impartió primero en la Sociedad de Instrucción pública en 1840 y luego en El Porvenir en 1848 son una obra inaugural (Moreno Yuste, 2006) con la que se consolida en español el término geología (Vernet Gines, 1989) un texto básico (Pelayo López, 1984) para conocer el estado de la geología española antes de la traducción que Ezquerra del Bayo publicara en 1847 de la obra de Lyell que, a juicio de Meléndez, abrió los caminos a la verdadera investigación paleontológica (Meléndez, 1947), interpretación clásica que Pelayo López matizó, subrayando que "al menos en lo que respecta a la paleontología, la cuestión fue mucho más compleja, y así se puede apreciar en las obras de los principales geólogos españoles de 1830 a 1870: Casiano del Prado,

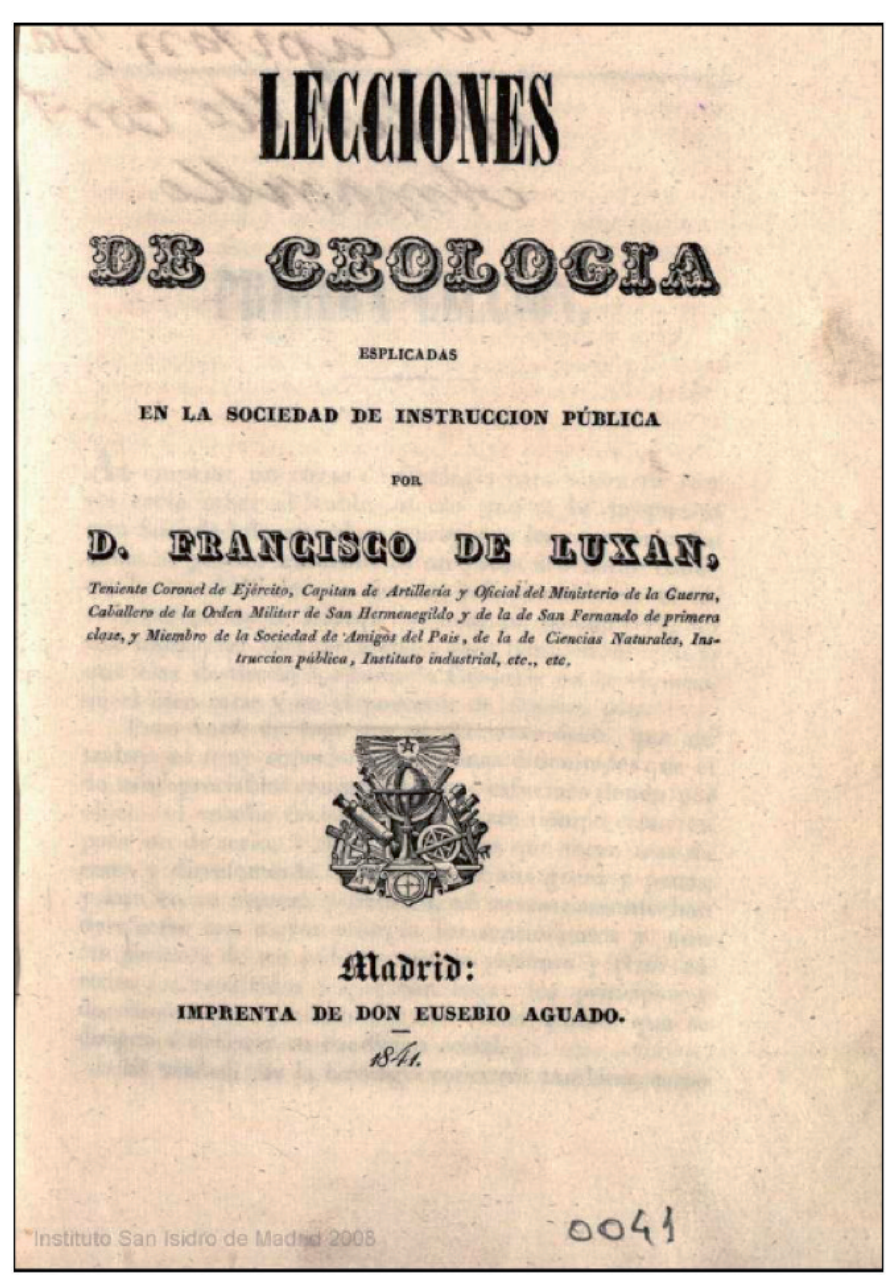

Figura 2. Luxán, F; Lecciones de Geología. Madrid 1841. Figure 2. Luxán, F.; Lecciones de Geología. Madrid 1841. 


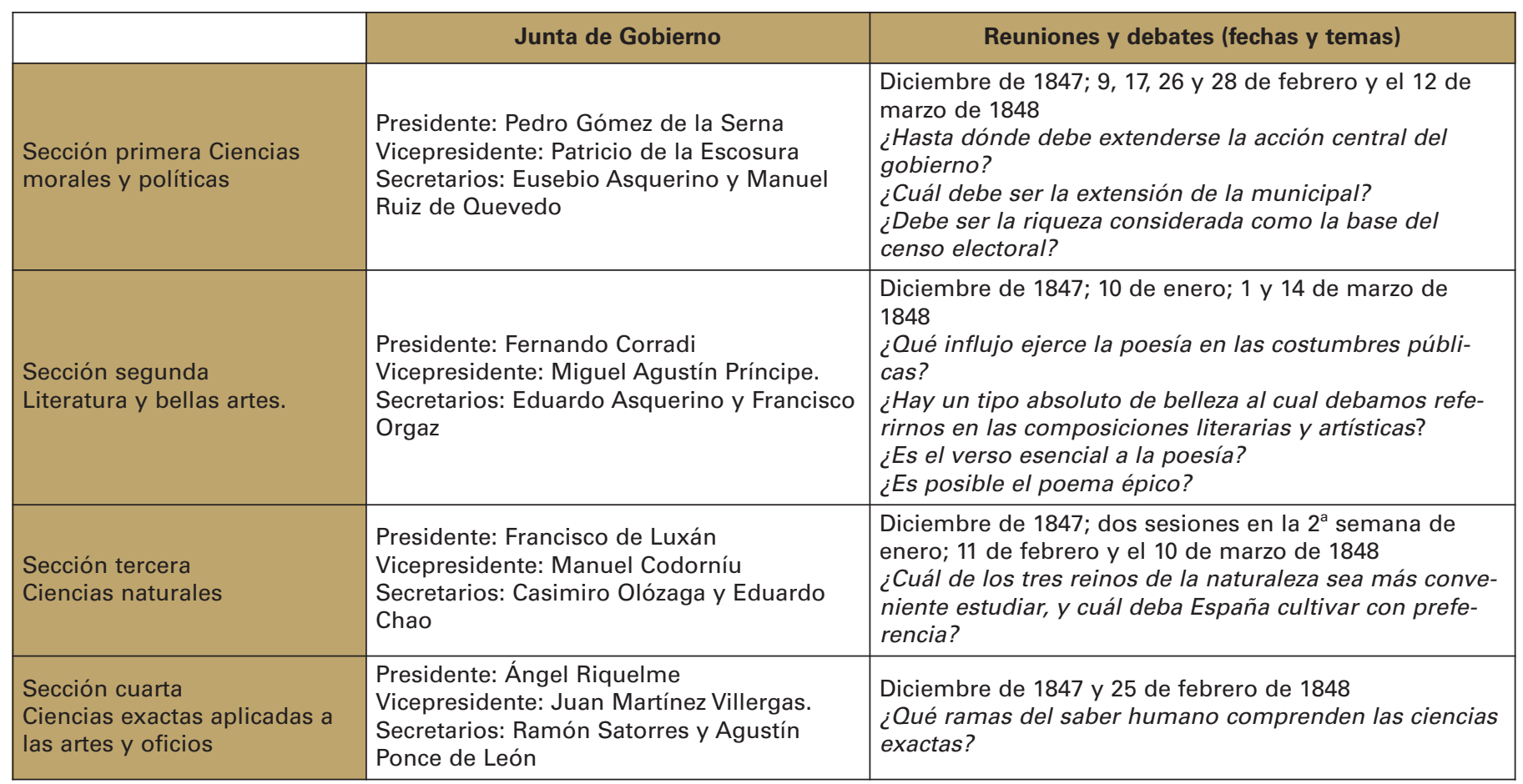

Tabla 6. Secciones de la sociedad El porvenir (1847-1848).

Table 6. Sections of society El porvenir (1847-1848).

Amar de la Torre, Vilanova y Piera, Luxán, y Ezquerra del Bayo" (Pelayo López, 1984).

En la de Instrucción Pública el curso de Luxán, comenzó el 8 de diciembre de 1840, y se estructuró en 19 sesiones, los martes a las siete de la tarde, que se corresponden con cada una de las 19 lecciones publicadas en 1841, y en El Porvenir sólo podrá impartir la primera lección, el 7 de febrero de 1848, a las ocho de la noche.

Luxán, tan buen hablista y tan erudito, en palabras de Martínez Villegas, director de EITio Camorra, inauguró su curso de geología, "definiendo la ciencia y demostrando la inmensa importancia de su estudio en el siglo actual. A grandes trazos y con la maestría y el profundo caudal de cocimientos que posee, describió su extensión inmensa y la relación íntima que la enlazan a las demás ciencias y continua el Eco del Comercio, e1 señor Luxán ha dado una muestra de lo que tiene acreditado ser; de profundo político, de aventajado naturalista, de consumado filósofo, de orador elocuente y de modelo de delicadeza y de finura. E1 público aplaudió tan bello discurso en que no sabemos nosotros qué elogiar más, si lo grande de los conocimientos o el brillante modo de exponerlos" (El Eco del Comercio 8 de febrero de 1848).

En sus lecciones es "de gran interés el debate que plantea sobre el origen de la vida, ya que expone las distintas teorías que estaban entonces vigentes: la bíblica, la catastrofista/transformista de Cuvier, el uniformismo de Lyell y, por último -con la que parce estar más de acuerdo-, la que sería una mezcla del creacionismo y el uniformismo catastrofista" (Boixereu Vila, 2016).

A Luxán, "dentro de su eclecticismo, se le puede considerar como un actualista no catastrofista. Aceptaba que las causas físicas que habían obrado en todas las épocas eran las mismas; lo que había sido diferente eran las circunstancias del medio" (Pelayo López, 1984).

Para El Espectador: "Estas lecciones que contienen los conocimientos admitidos, y según los últimos descubrimientos de la geología son utilísimas y necesarias á todas las personas que se dediquen á los ramos de minas, caminos, ingenieros civiles, militares, artilleros, oficiales de estado mayor, y aun á todo el que se ocupe de industria y del progreso de las artes mecánicas, la pericia de los españoles ha sido favorecida por la providencia en la constitución geológica de su suelo, y solo falta conocer las riquezas que este encierra y utilizarlas. La geología es la luz que debe guiar este conocimiento, y por lo tanto creemos hacer un servicio al país recomendando las lecciones del señor Luxán que muchas personas han oído explicar en el curso de 1840 al 1841 en la socie- 
José María de Luxán Meléndez, 2019. La geología en las sociedades de cultura... Boletín Geológico y Minero, 130 (2): $271-288$

dad de instrucción pública y á cuyo testimonio apelamos. El público de Madrid, que ha favorecido constantemente con su asistencia las explicaciones del señor Luxán, responderá de lo que decimos".

Luxán desataca el carácter científico de la Geología, que nacida con "Werner; desde 1814 ha corrido con pasos de gigante...en muchos países ha resonado el martillo del geólogo...Las observaciones de Hutton, (y las de sus coetáneos) Buch, Elie de Beaumont, Dufrenoy, Lyell, de la Beche, etc., han sentado bases ciertas y seguras; lo que era un sistema ha tomado el carácter y el rango de una ciencia" (Luxán Miguel-Romero F.,1841:7) $\mathrm{Y}$ presenta un programa que se extiende al estudio de la Astronomía, de la Atmosfera y de la Tierra, propiamente dicha.

Para Luxán con la "aplicación de los conocimientos geológicos a las artes y a la industria se abre un nuevo horizonte de investigaciones, utilísimo en el estado social de Europa...la geología es la luz que guía al minero...los pozos artesianos son imposible sin el conocimiento geológico...el ingeniero de caminos tiene en la geología un auxilio eficaz...la guerra necesita de la geología...la agricultura aprenderá en la geología“ (Luxán Miguel-Romero F.,1841:13-14

En la primera de las lecciones de Geología enfatiza que "debe entenderse que hablamos de ciencia y como geólogos, y que respetamos empero los principios y las creencias religiosas de nuestro país" (Luxán Miguel-Romero F.,1841:13) "Si hubo un tiempo en que se miraba con disgusto y aun con cierto horror religioso, las ciencias útiles al género humano, si lo hubo en que se reputaba como impío el estudio de la Geología, estos tiempos pasaron afortunadamente". (Luxán Miguel-Romero F.,1841:15) La advertencia llega ante un público progresista en un momento de crisis política en el que la libertad de imprenta jugaba un papel destacado (Castro Alín, 1998) y en un escenario en el que las posiciones de la iglesia se configuran como una de las principales líneas de fractura social y política.

Un terreno en el que por motivos religiosos (Sequeiros \& González Fabre, 2011), tan sólo cinco años antes, el decreto sobre impresión de libros que Javier de Burgos publicó en 1834, tras declarar en su artículo primero libre de censura todos los libros y papeles científicos, en el noveno, por el contrario, sometía a la Geología y a la Historia a censura previa (Real Decreto por el que se modifica el sistema de impresión, publicación y circulación de libros de 4 de enero de 1834. Gaceta de 7 de enero de 1834) y frente al que, en su Vindicación de la Geología, reaccionaría Casiano del Prado reclamando que los "progresos del espíritu humano ya exigen... que la geología se vea enteramente libre de ataduras entre nosotros, como lo está ya la astronomía y todas las ciencias y bástenos la vergüenza de ser los últimos que subsanemos esta clase de injusticias" (Prado, 1835).

\section{Las secciones de la sociedad El Porvenir}

El Porvenir contaba con cuatro secciones: ciencias morales y políticas; literatura y bellas artes; ciencias naturales y ciencias exactas aplicadas a las artes y oficios, que en diciembre de 1847 (El Clamor Público 18/12/1847) eligieron a sus Presidentes: en la primera, la de políticas a Pedro Gómez de la Serna, en la segunda, la literaria a Fernando Corradi, en la tercera, la de ciencias a Francisco de Luxán y en la cuarta la de exactas a Ángel Riquelme.

Junto a los Presidentes, cada sección nombrará también un Vicepresidente y dos Secretarios. En los debates de las secciones el público no podrá formar parte en la discusión. (El Espectador 9 de febrero de 1848, vuelve a señalarlo El Espectador el 17 de febrero, que indica que lo decidió la última Junta Directiva)

En la Sección de Ciencias Naturales junto a Luxán, será nombrado vicepresidente Manuel Codorníu (1788-1857), diputado en 1854 y secretarios Casimiro Olózaga (...-1866) miembro de la Academia Quirúrgica Matritense y Eduardo Chao Fernández (1822-1877) diputado, ministro de Fomento en 1873. La sección de ciencias naturales celebró al menos cinco sesiones, una en diciembre, dos en enero, una en febrero y otra en marzo de 1848.En las dos sesiones de enero discutió sobre ¿cuál de los tres reinos de la naturaleza sea más conveniente estudiar, y cuál deba España cultivar con preferencia?

En el debate participaron según El Espectador. Luxán, Codorníu, Chao, Esparrago y Segundo Flórez. Y se cruzaron dos planos de discusión. Uno de carácter teórico orientado a debatir, en el marco de las ciencias naturales, si se podía conceder más importancia a un reino que a otro, jerarquizar el estudio del reino mineral sobre el vegetal y este sobre el animal o a la inversa, cuestión a la que incorporó con palabras de El Espectador, el tema de la de la preexistencia o sucesora producción de los seres en la naturale$z a$, aspecto que al parecer animó el debate. Chao defendió que no se podía dar más importancia a un reino que a otro.

En segundo plano, hoy diríamos aplicado, se planteó qué campo científico era más conveniente desarrollar en España. Para Luxán se debía dar preferencia a la mineralogía. Y resume El Espectador "por lo que toca a los ramos de las ciencias naturales más dignas de atención en España., dividieron los votos entre, sí la agricultura y la mineralogía". 


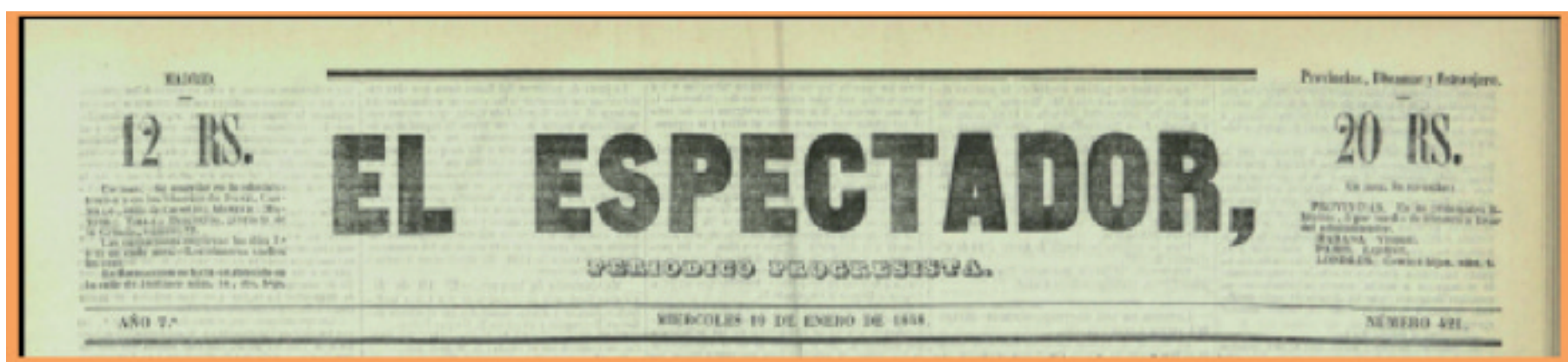

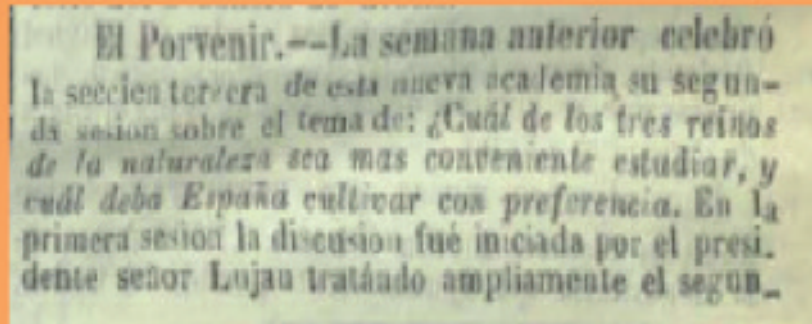

Figura 3. El Espectador, 19 de enero de 1848.

Figure 3. El Espectador January 19, 1848.

“En la primera sesión la discusión fue iniciada por el presidente señor Luxán tratando ampliamente el segundo extremo del lema con argumentos que le obligaban a dar la preferencia a la mineralogía; no faltaron, sin embargo, a la botánica y la zoología, con especialidad al hombre, partidarios entendidos como el señor Codorníu. En la segunda sesión el señor Chao levantó la discusión al terreno de la ciencia, al primer extremo del lema: su opinión era que no siéndonos conocidos todos los cuerpos de la naturaleza ni siéndolo bien los que hoy conocemos, parecía imposible conceder a ninguno la preferencia; que en su concepto, jamás podría determinase esta, porque do estremo del tema con argumentos que lo obilgaban $\mathbf{3}$ dar la preferencia a la mineralogia: no Galtaron, sin emhargo, a la botánira y. la zoolohia, con eaperialidad al liombre. partidarios eotendidos eomo el señor Codorní. En la segunda seecisn ef senor Chao levanto la discusion al terreno de la cieneia, al primer estremo del tema: su opinion era que no siéndonos canocidos todos los euerpos de la natnralera ni siéndolo bien los que hoy conocenos. parecia imposible conceder a vinguno la preforencia: que en $\$$ u eoncepto, janas podria determinarse sata, pongue cala especié desempeñaba en la organizacion tel globo fusciones propias euya importancia demostraban los trastoreos qte siguieran a sa falta: vimiendo a caneluir en que siendo los tres cuerpos especialmente necesarios, su estudio era igualmente importante, Las grandes euestiones con que bubo de roxarse el schor Chao, especialmente in de la preexistencia b sucesota produccion de los seres en la naturaleza, animó el debate que siguió sosteniendo prineipalmente eon el seînor Euparrago. Volví́ lueFo la discusion at terreno de la aplícacien el señor D. Segunde Florex con un discurso gue revelaba sus buenos estudios en esta parie de las ciencias, I eu la cconomia páblica: manifestó que la Espana está destinada por la naturalex a ser principalmente una nacion agricola sin dejar por eso de ser manafacturera; en cuya opinion disentia el senor Lujag, que, al reausumir la sesion pronencib un bello discurso en apoyo de la suya, aclarando at paso con sus Inces algunos puntos que se habian lalsealo en el debate. Esta sesion ha venido a probar tambien que el partido progresista cuenta en sus flas una brillante javentud bencbila de entusiasmo, de fe y de ciencia. cada especie desempeñaba en la organización del globo funciones propias cuya importancia demostraban los trastornos que siguieran a su falta; viniendo a concluir en que siendo los tres cuerpos especialmente necesarios, su estudio era igualmente importante, Las grandes cuestiones con que hubo de rozarse el señor Chao, especialmente la de la preexistencia o sucesora producción de los seres en la naturaleza, animó el debate que siguió sosteniendo principalmente con el señor Espárrago. Volvió luego la discusión al terreno de la aplicación el señor D. Segundo Flórez con un discurso que revelaba sus buenos estudios en esta parte de las ciencias, y en la economía 
pública: manifestó que la España está destinada por la naturaleza a ser principalmente una nación agrícola sin dejar por eso de ser manufacturera; en cuya opinión disentía el señor Luxán, que, al reasumir la sesión pronunció un bello discurso en apoyo de la suya, aclarando al paso con sus luces algunos puntos que se habían falseado en el debate. Esta sesión ha venido a probar también que el partido progresista cuenta en sus filas una brillante juventud henchida de entusiasmo, de fe y de ciencia" (Figura 3. El Espectador 19 de enero de 1848)

\section{Discusión/Conclusiones}

En las décadas centrales del XIX, junto al impulso de la ciencia desde el Estado es relevante la participación de la sociedad civil en el impulso de la ciencia mediante la recuperación de un modelo institucional ilustrado, que permitió la reconstrucción de corporaciones públicas de base asociativa como las Sociedades de Amigos del País, y las Academias científicas. Y además, mediante fórmulas asociativas de corte liberal, el desarrollo de nuevas o recuperadas sociedades culturales como el Ateneo, la Sociedad de Instrucción Pública, durante la regencia de Espartero, o El Porvenir en la década moderada.

Estas asociaciones culturales del reinado de Isabel II por sus actividades y por su organización, en un sentido amplio, pueden considerarse sociedades de cultura científica.

La vinculación entre política, cultura y ciencia, medida por la participación en el parlamento o en el gobierno de los científicos y de los profesores de las sociedades culturales o por la repercusión en la prensa de sus actividades es muy importante. Y además la vinculación partidista permite calificar como sociedades progresistas la Sociedad de Instrucción Pública, y la sociedad El Porvenir.

Las ciencias naturales, singularmente la enseñanza de la geología, tuvieron un papel destacado en las actividades y en la organización de las sociedades de cultura científica, tanto en el Ateneo como en las sociedades progresistas.

Además temas centrales como la preexistencia o sucesora producción de los seres en la naturaleza o la orientación de la política científica tuvieron una posición notable en la enseñanza y en los debates de las sociedades progresistas de cultura científica

La contribución de Francisco de Luxán en las sociedades progresistas de cultura científica es muy relevante, tanto como profesor de geología como por su posición en la Sección de Ciencias Naturales.

Durante el reinado de Isabel II el impacto de las sociedades de cultura científica, medido por su continuidad o discontinuidad es muy desigual. A diferencia del Ateneo, la huella en la cultura científica de las sociedades progresistas estuvo atenuada por su escasa duración, lo que no afecta al peso de los científicos progresistas que se deduce sobre todo de su contribución, desde el Estado, al diseño y desarrollo de una política pública para la ciencia y para la sociedad industrial.

\section{Agradecimientos}

Este artículo deriva de una de los temas abiertos en la Tesis doctoral que dirigida por Blanca Olías de Lima defendí en 2015 en la Universidad Complutense de Madrid. Una primera versión fue presentada en el coloquio que sobre Geología y Política. Una política para la ciencia en la España liberal, se celebró en Geoforo del Colegio de Geólogos de España el 24 de abril de 2017, en el que participe junto a Salvador Ordoñez y ha sido mejorada con las sugerencias de los asistentes, y también con los comentarios críticos de Ester Boixareu, Luis de Luxán, Leoncio LópezOcón y Blanca Olías de Lima a los que agradezco sus acertadas y detalladas observaciones, aunque sólo soy yo responsable del resultado. La traducción al Inglés de la versión abreviada ha sido revisada por Guillermo de Luxán.

\section{Referencias}

Boixereu Vila, E. 2016. La dimensión científica del geólogo Francisco de Luxán. En Milicia y Geología. Francisco de Luxán (págs. 60-70). Madrid : Ministerio de Defensa y Ministerio de Economía y Competitividad.

Burgueño, J. 1994. La geografía entesa per un liberal. Un text inèdit de Fermín Caballero (1840). Treballs de la Societat Catalana de Geografia. $n^{\circ} 38$ vol IX, 57-68.

Castro Alín, D. 1998. Los males de la imprenta. Política y libertad de prensa en una sociedad dual. Madrid: Centro de Investigaciones Sociológicas.

Elorza, A. 1987. Estudio preliminar. En J. M. López, Curso Político Constitucional. Madrid: Centro de Estudios Políticos y Constitucionales.

Garrorena Morales, A. 1974. El Atenero de Madrid y la teoria de la monarquia liberal, 1836-1847. Madrid: Instituto de Estudios Políticos.

Llorente, M. 1836. Resumen de las Memorias de la Academia de Ciencias Naturales. 1836. Madrid: Imprenta del Colegio de Sordomudos.

López Piñero, J. (Ed.). 1992. La Ciencia en la España del siglo XIX. Madrid: Marcial Pons.

López, J. 1840. Curso político-constitucional. Madrid 1987: Centro de estudios constitucionales. 
López-Ocón Cabrera, L. 2006. Ciencia y progreso durante la época bajoisabelina (1854-1868). En M. Súarez Cortina (Ed.), La redención del pueblo. La cultura progresista en la españa liberal (págs. 315-346). Santandder: Universdad de Cantabria.

López-Ocón Cabrera, L. 2003. Breve historia de la ciencia española. Madrid: Alianza editorial.

Luxán Meléndez, J. M. 2016. Los políticos del progreso. Científicos en el gobierno y en el Partido Progresista (1833-1868). Cuadernos de Gobierno y Administración Pública 3-1.

Luxán Meléndez, J. M. 2016. Una política para la ciencia en el reinado de Isabel II. La contribución de Francisco de Luxán y su relación con la Comisión del Mapa Geológico. Madrid: Centro de Estudios Políticos y Constitucionales.

Luxán Meléndez, J. M. 2016. Continuidad y discontinuidad de los progresistas extremeños en el reinado de Isabel II. Revista de Estudios Extremeños Tomo LXXII, Número III , 1785-1824.

Luxán Miguel-Romero, F. 1841. Lecciones de Geología esplicadas en la Sociedad de Instrucción pública. Madrid: Imprenta de Don Eusebio Aguado.

Madoz, P. 1845-1850. voz Madrid. En P. Madoz, Diccionaro Geográfico-Estadístico-histórico de España y sus posesiones de ultramar. Tomo $X$. Madrid.

Meléndez, B. 1947. Tratado de paleontología Tomo I. Madrid (1998): Consejo Superior de Investigaciones Científicas

Monreal, J. M. 1839. Memoria leida en el Ateneo científico de Madrid, en la Junta general de 21 de diciembre de 1839. Madrid: Imprenta del colegio nacional de SordoMudos.

Montero, Á. 2003. La paleontología y sus colecciones desde el Real Gabinete de Historia Natural al Museo Nacional de Ciencias Naturales. Madrid: Consejo Superior de Investigaciones Científicas.

Moreno Yuste, J. 2006. Las ciencias naturales (Química y mineralogía) y el Colegio de Artillería de Segovia:18391928. Segovia: Biblioteca de Ciencia y Artilleria.

Muro, J. I., Nadal, F., \& Urteaga, L. 1996. Geografía, estadítica y catastro en España (1856-1870). Barcelona: Ediciones del Serbal.

Pan-Montojo, J. 2014. Progreso material, Fomento y Libertas: La economía en las culturas políticas del peiodo Isabelino. En M. Cruz Romeo, \& M. Sierra (Edits.), La España Liberal 1833-1874 (págs. 51-87). Madrid: Marcial Pons Ediciones de Historia y Prensas de la Universidad de Zaragoza.

Pelayo López, F. 1984. Catastrofismo y Actualismo en España. LLULL, vol 7 , 47-68.

Prado, C. 1835. Vindicación de la Geología. Madrid: Imprenta de D. Eusebio Aguado.

Quintanilla Fisac, M. Á. 2010. La ciencia y la cultura científica. Artefactos, vol. $3 n^{\circ} 1,31-48$.

Rábano Gutierrez del Arroyo, I. 2015. Los Cimientos de la Geología. La Comisión del Mapa Geológico de España (1849-1910). Madrid: Instituto Geológico y Minero de España.

Rabano Gutierrez del Arroyo, I. 2016. Francisco de Luxán, prsidente de la Comisión del Mapa geológico. En Milicia y geología. Francisco de Luxán (págs. 42-52). Madrid: Ministerio de Defensa y Ministerio de Econía y Competitividad.

Sequeiros, L., \& González Fabre, M. 2011. Filosofía de Geología y Religión: La vindicación de la geología de Casiano del Prado (1835). Pensamiento Vol 67, 963-1005.

Recibido: febrero 2018

Revisado: mayo 2018

Aceptado: septiembre 2018

Publicado: junio 2019 